\title{
CLOSURE SETS OF FUNCTIONS AND A HIERARCHY OF FILTERS
}

\author{
ROBERT MIGNONE
}

(Communicated by Andreas R. Blass)

\begin{abstract}
This paper presents a transfinite extension of a filter generating closure property of functions. One consequence of this extension is a hierarchy of filters which coincide with filters generated by a directed set type closure property. At each level of this hierarchy a progressively stronger notion of normality is satisfied. Ideals with this stronger notion of normality each have a corresponding saturation characterization.
\end{abstract}

Normality for filters on $P_{\kappa} \lambda$ can be defined in terms of regressive functions from $P_{\kappa} \lambda$ into $\lambda$. It is well known that an equivalent definition for normality can be given in terms of regressive functions from $P_{\kappa} \lambda$ into $P_{\omega} \lambda$. Likewise, the minimal normal filter can be characterized as the filter generated by sets closed under functions from $\lambda \times \lambda$ into $P_{\kappa} \lambda$ and an equivalent characterization can be made using functions from $P_{\omega} \lambda$ into $P_{\kappa} \lambda$. What if these two notions were extended to functions from $P_{\kappa} \lambda$ into $P_{\delta} \lambda$ and functions from $P_{\delta} \lambda$ into $P_{\kappa} \lambda$ respectively, for appropriate $\delta<\kappa$ ? This question leads to the concepts and results in the present paper.

Standard notation is used throughout. Let $\kappa$ be a regular cardinal. If $\lambda \geq \kappa$ is a cardinal with $c f(\lambda) \geq \kappa$,

$$
P_{\kappa} \lambda=\{x \subset \lambda:|x|<\kappa\} .
$$

Given $x \in P_{\kappa} \lambda$, let $\hat{x}=\left\{y \in P_{\kappa} \lambda: x \subseteq y\right\}$. If $F$ is a filter on $P_{\kappa} \lambda, F^{*}$ is its dual ideal and vice versa, and

$$
F^{+}=\left\{A \subseteq P_{\kappa} \lambda: A \notin F^{*}\right\} .
$$

The final section filter is given by

$$
F S F_{\kappa \lambda}=\left\{A \subseteq P_{\kappa} \lambda: \hat{x} \subseteq A \text { for some } x \in P_{\kappa} \lambda\right\} .
$$

Filters over $P_{\kappa} \lambda$ extending $F S F_{\kappa \lambda}$ are called fine. And $A \subseteq P_{\kappa} \lambda$ is said to be unbounded if $A \cap \hat{x} \neq \varnothing$ for all $x \in P_{\kappa} \lambda$. For the extent of this paper, all filters

Received by the editors April 20, 1990 and, in revised form, September 14, 1990; the results contained in this paper were presented in University Park, Pennsylvania, April 1990, during the spring meeting of the American Mathematical Society.

1980 Mathematics Subject Classification (1985 Revision). Primary 04A20.

This research was partially supported by a College of Charleston Research Grant and a Southeastern Regional Education Board Grant. The author also wishes to express his appreciation to MSRI, where this research was carried out. 
(ideals) will be $\kappa$-complete extensions of $F S F_{\kappa \lambda}\left(F S F_{\kappa \lambda}{ }^{*}\right)$. A set $C \subseteq P_{\kappa} \lambda$ is said to be closed under chains, if whenever $\left\{x_{\gamma}: \gamma<\delta\right\} \subset C$ where $x_{\gamma} \subseteq x_{\gamma^{\prime}}$, when $\gamma<\gamma^{\prime}<\delta<\kappa$, then $\bigcup\left\{x_{\gamma}: \gamma<\delta\right\} \in C$. The closed unbounded filter is given by

$$
C F_{\kappa \lambda}=\left\{A \subseteq P_{\kappa} \lambda: C \subseteq A \text { for some closed unbounded set } C\right\} .
$$

Let

$$
\bar{\delta}=\inf \left\{\delta \leq \kappa: \exists \gamma<\kappa \text { and } \gamma^{<} \delta \geq \kappa\right\} .
$$

Definition 1. For $\delta$ regular, let $v: P_{\delta} \lambda \longrightarrow P_{\kappa} \lambda$ and

$$
C(v)=\left\{x \in P_{\kappa} \lambda: y \in \mathbf{P}_{\delta} x \Longrightarrow v(y) \subset x\right\} .
$$

If $x \in C(v)$, then $x$ is said to be closed under $v$ and $C(v)$ is called the closure set of $v$.

Definition 2. Let $\delta$ be regular and $\delta<\bar{\delta}$. Given $v: P_{\delta} \lambda \longrightarrow P_{\kappa} \lambda$, define $v^{\prime}$ as follows: for $x \in P_{\kappa} \lambda$, let

$$
v_{1}(x)=\bigcup\left\{v(y): y \in P_{\delta} x\right\} \cup x ;
$$

and for $1<\gamma<\delta$,

$$
v_{\gamma}(x)=\bigcup\left\{v(y): y \in P_{\delta}\left(\bigcup\left\{v_{\xi}(x): \xi<\gamma\right\}\right)\right\} .
$$

Set

$$
v^{\prime}(x)=\bigcup_{\gamma<\delta} v_{\gamma}(x) .
$$

Definition 3. For a regular $\delta<\kappa, D \subseteq P_{\kappa} \lambda$ is said to be $\delta$-directed, if whenever $d \subset D$ and $|d|<\delta$, there exists a $y \in D$ such that $\bigcup d \subseteq y$. And $C \subseteq P_{\kappa} \lambda$ is said to be $\delta$-dclosed, if whenever $D \subset C$ such that $|D|<\kappa$ and $D$ is $\delta$-directed, then $\bigcup D \in C$.

Theorem 4. Let $\delta<\bar{\delta}$ be regular. Suppose that $v: P_{\delta} \lambda \longrightarrow P_{\kappa} \lambda$ and $w$ : $P_{\delta} \lambda \longrightarrow P_{\kappa} \lambda$. Then

(1) $C(v)=C\left(v^{\prime}\right)$;

(2) $\forall x \in P_{\kappa} \lambda\left(x \subseteq v^{\prime}(x) \wedge v^{\prime}(x) \in C\left(v^{\prime}\right)\right)$;

(3) $\forall x \in P_{\delta} \lambda\left(v^{\prime}(x)=\bigcup\left\{v^{\prime}(y): y \in P_{\delta}\left(v^{\prime}(x)\right)\right\}\right)$;

(4) $\forall x, y \in P_{\delta} \lambda\left(\left(v^{\prime}(x) \cap v^{\prime}(y)\right) \in C\left(v^{\prime}\right)\right)$;

(5) $\forall x \in P_{\delta} \lambda\left(C(w) \subset C(v) \leftrightarrow v^{\prime}(x) \subset w^{\prime}(x)\right)$;

(6) $\forall x \in P_{\delta} \lambda\left(v^{\prime}(x)\right.$ is the smallest set closed under $v$, containing $\left.x\right)$;

(7) $C\left(v^{\prime}\right)=\left\{\bigcup v^{\prime}[x]: x \in P_{\kappa} \lambda\right\}$, where $v^{\prime}[x]=\left\{v^{\prime}(y): y \in P_{\delta} x\right\}$;

(8) $v^{\prime}[x]$ is $\delta$-directed.

Proof.

(1) $C\left(v^{\prime}\right) \subset C(v)$ since $\forall x \in P_{\delta} \lambda\left(v(x) \subset v^{\prime}(x)\right)$. Next, given $x \in C(v)$, let $y \in P_{\delta} x$. Then by induction on $\gamma<\delta$,

$$
v_{1}(y)=\bigcup\left\{v(z): z \in P_{\delta} y\right\} \cup y \subset x ;
$$

and

$$
v_{\gamma}(y)=\bigcup\left\{v(z): z \in P_{\delta}\left(\bigcup\left\{v_{\gamma^{\prime}}(y): \gamma^{\prime}<\gamma\right\}\right)\right\} \subset x,
$$


since $\forall \gamma^{\prime}<\gamma\left(v_{\gamma^{\prime}}(y) \subset x\right)$, by the induction hypothesis. Hence, $v^{\prime}(y) \subset x$ and $x \in C\left(v^{\prime}\right)$.

(2) Clearly, $x \in P_{\kappa}\left(v^{\prime}(x)\right)$. And if $y \in P_{\delta}\left(v^{\prime}(x)\right)$, then $|y|<\delta$, so $y \subset v_{\gamma}(x)$ for some $\gamma<\delta$. Hence $v(y) \in v_{\gamma+1}(x)$.

(3) Let $\alpha \in v^{\prime}(x)$. Then $\alpha \in v^{\prime}(\{\alpha\}) \subset \bigcup\left\{v^{\prime}(y): y \in P_{\delta}\left(v^{\prime}(x)\right)\right\}$. And if $\alpha \in \bigcup\left\{v^{\prime}(y): y \in P_{\delta}\left(v^{\prime}(x)\right)\right\}$, then $\alpha \in v^{\prime}(y) \subset v^{\prime}(x)$ by (2).

(4) This holds since $v^{\prime}(x)$ and $v^{\prime}(y)$ are in $C(v)$ by (2).

(5) For the first direction, $x \in P_{\delta}\left(w^{\prime}(x)\right)$ and $w^{\prime}(x) \in C(v)=C\left(v^{\prime}\right)$ by (1). Hence, $v^{\prime}(x) \subset w^{\prime}(x)$. For the remaining direction, if $x \in C(w)$ and $y \in P_{\delta} x$, then $v^{\prime}(y) \subset w^{\prime}(y) \subset x$. Hence, $x \in C(v)$.

(6) Given $x \in P_{\delta} \lambda$, let $z \in C(v)$ such that $x \subset z$. Then $v^{\prime}(x) \subset z$.

(7) Let $x \in C\left(v^{\prime}\right)$. Then $x=\bigcup v^{\prime}[x]$. This is so, since given $\alpha \in x$, then $\alpha \in v^{\prime}(\{\alpha\}) \in\left\{v^{\prime}(y): y \in P_{\delta} x\right\}$. And if $\alpha \in \bigcup v^{\prime}[x]$, then $\alpha \in v^{\prime}(y)$ and $y \in P_{\delta} x$, so $\alpha \in x$. Next, let $y \in P_{\delta}\left(\bigcup v^{\prime}[x]\right)$. Then $y \subset \bigcup\left\{v^{\prime}(z): z \in P_{\delta} x\right\}$. Let $z_{\alpha} \in P_{\delta} x$ such that $\alpha \in v^{\prime}\left(z_{\alpha}\right)$ for $\alpha \in y$. So $y \subset \bigcup\left\{v^{\prime}\left(z_{\alpha}\right): \alpha \in y\right\} \subset$ $v^{\prime}\left(\bigcup\left\{z_{\alpha}: \alpha \in y\right\}\right)$. But $v^{\prime}\left(\bigcup\left\{z_{\alpha}: \alpha \in y\right\} \in C\left(v^{\prime}\right)\right.$; hence $v^{\prime}(y) \subset \bigcup v^{\prime}[x]$.

(8) Let $d=\left\{v^{\prime}\left(y_{\gamma}\right): \gamma<\xi\right\} \subset v^{\prime}[x]$, where $y_{\gamma} \in P_{\delta} x$ and $\xi<\delta$. Since $\delta$ is regular, $\left|\bigcup\left\{y_{\gamma}: \gamma<\xi\right\}\right|<\delta$, and hence $\bigcup\left\{y_{\gamma}: \gamma<\xi\right\} \in P_{\delta} x$. By (3), $\bigcup d \subset v^{\prime}\left(\bigcup\left\{y_{\gamma}: \gamma<\xi\right\}\right)$.

The next definition strengthens the notion of normality for filters on $P_{\kappa} \lambda$.

Definition 5. A filter $F$ on $P_{\kappa} \lambda$ is $\delta$-normal, if whenever $f: P_{\kappa} \lambda \longrightarrow P_{\delta} \lambda$ such that $A \in F^{+}$and $\forall x \in A\left(f(x) \in P_{\delta} x\right)$, then $\exists B \subseteq A$, and $y \in P_{\delta} \lambda$ such that $B \in F^{+}$and $f(x)=y \forall x \in B$.

As in the case for normality, $\delta$-normality can be characterized by a type of diagonal intersection. The symbol $\Delta^{\delta}$ will be used to indicate the diagonal intersection of a collection of subsets of $P_{\kappa} \lambda$ indexed over $P_{\delta} \lambda$.

Theorem 6. A filter $F$ on $P_{\kappa} \lambda$ is $\delta$-normal if and only if whenever $\left\{C_{y}: y \in\right.$ $\left.P_{\delta} \lambda\right\} \subset F$ then

$$
\triangle^{\delta}\left\{C_{y}: y \in P_{\delta} \lambda\right\}=\left\{x \in P_{\kappa} \lambda: y \in P_{\delta} x \Longrightarrow x \in C_{y}\right\} \in F .
$$

Proof. The usual argument adapts to this situation, see [Je1].

In [Me], Menas showed that the filter generated by closure sets of functions $v: P_{\omega} \lambda \longrightarrow P_{\kappa} \lambda$ is the same as the closed unbounded filter, $C F_{\kappa \lambda}$, on $P_{\kappa} \lambda$. In the same paper, Menas mentions and uses the fact that the filter generated by closure sets of functions $v: P_{\omega} \lambda \longrightarrow P_{\kappa} \lambda$ is the same as the filter generated by closure sets of functions $v: \lambda \times \lambda \longrightarrow P_{\kappa} \lambda$. This fact has proved useful in obtaining results involving the closed unbounded filter on $P_{\kappa} \lambda$. In particular, Carr used it to prove that $C F_{\kappa \lambda}$ is the minimal normal filter on $P_{\kappa} \lambda$, see [Ca]; Johnson used it to get results on $M$-ideals on $P_{\kappa} \lambda$, see [Jo]. Also in [Me], is the slightly hidden fact that the filter generated by the closure sets of functions $v$ : $\lambda \longrightarrow P_{\kappa} \lambda$ is the same as $S C F_{\kappa \lambda}$, the strongly closed unbounded filter (which is the filter generated by unbounded subsets of $P_{\kappa} \lambda$ closed under arbitrary unions of size less than $\kappa$ ). In [Mi], this fact and a theorem similiar to Theorem 4 for $v: \lambda \longrightarrow P_{\kappa} \lambda$ are used to establish results about quasinormal filters on $P_{\kappa} \lambda$.

In what follows, a general theory is developed for filters generated by $v$ : $P_{\delta} \lambda \longrightarrow P_{\kappa} \lambda$, where $\delta<\bar{\delta}$ is regular. What emerges is a strict linear hierarchy 
of filters (ideals), each coinciding with a corresponding $\delta$-closed unbounded filter and each satisfying a progressively stronger normality property, namely $\delta$-normality.

Definition 7. Let $\delta<\bar{\delta}$ be regular. The closure set filter is given by

$$
F_{\kappa \lambda}^{\delta}=\left\{A \subset P_{\kappa} \lambda: \exists v: P_{\delta} \lambda \longrightarrow P_{\kappa} \lambda \text { and } C(v) \subseteq A\right\} .
$$

Theorem 8. $F_{\kappa \lambda}^{\delta}$ is a $\delta$-normal, $\kappa$-complete fine filter on $P_{\kappa} \lambda$.

Proof. The verification that $F_{\kappa \lambda}^{\delta}$ is a $\kappa$-complete, fine filter is routine. Let

$$
\left\{C\left(v_{y}\right): y \in P_{\delta} \lambda\right\} \subset F_{\kappa \lambda}^{\delta} \text {. }
$$

Define $v(z)=\bigcup\left\{v_{y}^{\prime}(z): y \in P_{\delta} z\right\}$. Let $x \in C(v), y \in P_{\delta} x$ and $z \in P_{\delta} x$. Now

$$
v(z \cup y)=\bigcup\left\{v_{t}^{\prime}(z \cup y): t \in P_{\delta}(z \cup y)\right\} .
$$

This gives $v_{y}(z) \subset v_{y}^{\prime}(z \cup y) \subset v(z \cup y) \subset x$. The first inclusion follows from the construction of $v_{y}^{\prime}$ from $v_{y}$, the second inclusion from the definition of $v$ and the third from the fact that $x \in C(v)$.

Definition 9. Let $\delta<\bar{\delta}$, be regular.

$$
D C F_{\kappa \lambda}^{\delta}=\left\{A \subset P_{\kappa} \lambda: \exists C \subseteq A \text { where } C \text { is } \delta \text {-dclosed unbounded }\right\} .
$$

Note. If $C \subseteq P_{\kappa} \lambda$ is $\delta$-dclosed unbounded, then $C$ is closed under unions of chains from $C$ of cofinal length greater than or equal to $\delta$ (call this $\delta$-closed). The converse does not appear to yield to Solovay's method, see [Mag]. It would be interesting to determine whether or not a set is $\delta$-dclosed unbounded if and only if it is $\delta$-dclosed unbounded. Or, if the filter generated by $\delta$-dclosed unbounded sets is denoted by $C F_{\kappa \lambda}^{\delta}$, then $D C F_{\kappa \lambda}^{\delta} \subset C F_{\kappa \lambda}^{\delta}$, but does equality hold?

Both $D C F_{\kappa \lambda}^{\delta}$ and $C F_{\kappa \lambda}^{\delta}$ are $\kappa$-complete, fine, $\delta$-normal filters on $P_{\kappa} \lambda$. For $D C F_{\kappa \lambda}^{\delta}$ it is a consequence of the next theorem and for $C F_{\kappa \lambda}^{\delta}$ the verification is routine and will be omitted.

Theorem 10. For $\delta<\bar{\delta}$, regular, $F_{\kappa \lambda}^{\delta}=D C F_{\kappa \lambda}^{\delta}$.

Proof. Given $v: P_{\delta} \lambda \longrightarrow P_{\kappa} \lambda$, let $D \subset C(v)$ be $\delta$-directed, where $|D|<\kappa$. Let $x \in P_{\delta}(\bigcup D)$. For each $\alpha \in x$, there exists a $x_{\alpha} \in D$ such that $\alpha \in x_{\alpha}$. There exists a $y \in D$ such that $\bigcup_{\alpha \in x} x_{\alpha} \subset y$. So $x \in P_{\delta} y$. This gives $v(x) \subset$ $y \subset \bigcup D$. Note, $C(v)$ is unbounded by parts (1) and (2) of Theorem 4. Hence $F_{\kappa \lambda}^{\delta} \subset D C F_{\kappa \lambda}^{\delta}$.

Next, assume $A \subset P_{\kappa} \lambda$ is closed under $\delta$-directed subsets of cardinality less than $\kappa$, and unbounded. Define $v_{\gamma}: P_{\delta} \lambda \longrightarrow P_{\kappa} \lambda$, for $\gamma<\delta$, as follows: for each $y \in P_{\delta} \lambda$,

$$
\begin{array}{ll}
v_{1}(y) \in A, & \text { where } y \subset v_{1}(y) ; \\
v_{\gamma}(y) \in A, & \text { where } \bigcup\left\{v_{\gamma^{\prime}}(z): \gamma^{\prime}<\gamma \text { and } z \subset y\right\} \subset v_{\gamma}(y) .
\end{array}
$$

Claim. For every $x$ in $P_{\kappa} \lambda, D_{x}=\left\{v_{\gamma}(y): \gamma<\delta\right.$ and $\left.y \in P_{\delta} x\right\}$ is a $\delta$-directed subset of $P_{\kappa} \lambda$, of cardinality less than $\kappa$. 
Proof of claim. Since $\delta<\bar{\delta},\left|D_{x}\right|<\kappa$. So let

$$
\left\{v_{\gamma_{\alpha}}\left(y_{\alpha}\right): \alpha<\delta^{\prime}\right\} \subset D_{x}, \quad \text { where } \delta^{\prime}<\delta .
$$

Setting $\gamma=\sup \left\{\gamma_{\alpha}: \alpha<\delta^{\prime}\right\}+1$ and $y=\bigcup_{\alpha<\delta^{\prime}} y_{\alpha}$, gives $\gamma<\delta$ and $y \in P_{\delta} x$. By construction

$$
\bigcup\left\{v_{\gamma_{\alpha}}\left(y_{\alpha}\right): \alpha<\delta^{\prime}\right\} \subset v_{\gamma}(y) \in D_{x} .
$$

This proves the claim.

Define $v: P_{\delta} \lambda \longrightarrow P_{\kappa} \lambda$ by $v(y)=\bigcup D_{y}$. Let $x \in C(v)$. The construction of $v_{y}$ and $D_{x}$ yields $x \subset \bigcup D_{x}$. And if $\alpha \in \bigcup D_{x}$, then $\alpha \in v_{y}(y)$ for some $y \in P_{\delta} x$. But

$$
v_{y}(y) \subset \bigcup D_{y}=v(y) \subset x .
$$

Hence, $\bigcup D_{x} \subset x$. Since $A$ is closed under $\delta$-directed sets of cardinality less than $\kappa$, by the claim, $\bigcup D_{x} \in A$. Hence $x \in A$ and $C(v) \subset A$. This shows $D C F_{\kappa \lambda}^{\delta} \subset F_{\kappa \lambda}^{\delta}$.

The next theorem shows that the $F_{\kappa \lambda}^{\delta}$ form a strictly increasing hierarchy.

Theorem 11. $F_{\kappa \lambda}^{\delta} \subsetneq F_{\kappa \lambda}^{\delta^{+}}$, for all regular $\delta<\bar{\delta}$.

Proof. Define $v: P_{\delta^{+}} \lambda \longrightarrow P_{\kappa} \lambda$ as follows:

$$
v(x)=\{\sup x\} .
$$

The result will follow from Theorem 10 once it is demonstrated that no unbounded subset of $C(v)$ can be closed under $\delta$-directed sets. Let $A \subset C(v)$ be an unbounded subset. Construct $\left\{\alpha_{\gamma}: \gamma<\delta\right\}$ and $\left\{x_{\gamma}: \gamma<\delta\right\}$ as follows: Let $\alpha_{1} \in \lambda$, choose $x_{1} \in A$ such that $\alpha_{1} \in x_{1}$. If $\xi<\gamma$ and we have $\alpha_{\xi} \in \lambda$ and $x_{\xi} \in A$ defined, choose

$$
\alpha_{\gamma}>\sup \left\{\bigcup x_{\xi}: \xi<\gamma\right\} .
$$

Now choose $x_{\gamma} \in A$ such that $\alpha_{\gamma} \in x_{\gamma}$ and $x_{\xi} \subset x_{\gamma}$ when $\xi<\gamma$. This shows that

$$
\begin{gathered}
\left\{x_{\gamma}: \gamma<\delta\right\} \quad \text { is } \delta \text {-directed, } \\
\left|\left\{x_{\gamma}: \gamma<\delta\right\}\right|<\kappa,
\end{gathered}
$$

and

$$
\left\{x_{\gamma}: \gamma<\delta\right\} \subset A \text {. }
$$

Suppose $\bigcup\left\{x_{\gamma}: \gamma<\delta\right\} \in A$. Consider $x=\left\{\alpha_{\gamma}: \gamma<\delta\right\}$. Then

$$
x \in P_{\delta^{+}}\left(\bigcup\left\{x_{\gamma}: \gamma<\delta\right\}\right)
$$

and

$$
\bigcup\left\{x_{\gamma}: \gamma<\delta\right\} \in C(v)
$$

since $A \subset C(v)$. So

$$
v(x)=\{\sup x\} \subset \bigcup\left\{x_{\gamma}: \gamma<\delta\right\} .
$$

But clearly $\sup x \notin \bigcup\left\{x_{\gamma}: \gamma<\delta\right\}$, a contradiction.

The next set of definitions and theorem will be used to establish that, at each level of the hierarchy, $F_{\kappa \lambda}^{\delta}$ is the minimal $\delta$-normal filter on $P_{\kappa} \lambda$. 
Definition 12. For any filter $F$ on $P_{\kappa} \lambda$, denote

$$
\triangle F=\left\{A \subset P_{\kappa} \lambda: \exists\left\{A_{\alpha}: \alpha<\lambda\right\} \subset F \text { and } \triangle_{\alpha<\lambda} A_{\alpha} \subseteq A\right\} .
$$

In [Ca], Carr determined that for every filter $\mathrm{F}$ on $P_{\kappa} \lambda$ extending $F S F_{\kappa \lambda}$ :

(1) $F \subseteq \triangle F$, where equality holds if $F$ is normal;

(2) $\triangle F S F_{\kappa \lambda}=S C F_{\kappa \lambda}=\left(F_{\kappa \lambda}^{2}\right)$;

(3) $\triangle \triangle F S F_{\kappa \lambda}=\triangle S C F_{\kappa \lambda}=C F_{\kappa \lambda}\left(=F_{\kappa \lambda}^{3}=F_{\kappa \lambda}^{\omega}\right)$.

Definition 13. For any filter $F$ on $P_{\kappa} \lambda$, let

$$
\triangle^{\delta} F=\left\{A \subseteq P_{\kappa} \lambda: \exists\left\{A_{y}: y \in P_{\delta} \lambda\right\} \subset F \text { and } \triangle^{\delta}\left\{A_{y}: y \in P_{\delta} \lambda\right\} \subset A\right\} .
$$

Theorem 14. For regular $\delta<\bar{\delta}$,

(1) $\triangle^{\delta} F S F_{\kappa \lambda}=F_{\kappa \lambda}^{\delta}$;

(2) $\Delta^{\delta} F_{\kappa \lambda}^{\xi}=F_{\kappa \lambda}^{\delta}$ for all regular $\xi<\delta$.

Proof. For the proof of part one, let $C \in \triangle^{\delta} F S F_{\kappa \lambda}$. Without loss of generality, assume

$$
C=\triangle^{\delta}\left\{\hat{x}_{y}: y \in P_{\delta} \lambda\right\},
$$

where $x_{y} \in P_{\kappa} \lambda$ for $y \in P_{\delta} \lambda$. Define $v_{y}: P_{\delta} \lambda \longrightarrow P_{\kappa} \lambda$ by $v_{y}(z)=x_{y}$. Given

$$
x \in \Delta^{\delta}\left\{C\left(v_{y}\right): y \in P_{\delta} \lambda\right\} \in F_{\kappa \lambda}^{\delta} .
$$

If $y \in P_{\delta} x$, then $x \in C\left(v_{y}\right)$. So $x_{y}=v_{y}(z) \subset x$ for every $z \in P_{\delta} x$. Hence $x \in \hat{x}_{y}$, giving $x \in C$ and therefore $C \in F_{\kappa \lambda}^{\delta}$.

Next, let $v: P_{\delta} \lambda \longrightarrow P_{\kappa} \lambda$ and consider $C(v)$. For each $y \in P_{\delta} \lambda$, let $x_{y} \in C(v)$ such that $y \subset x_{y}$. Given $x \in \Delta^{\delta}\left\{\hat{x}_{y}: y \in P_{\delta} \lambda\right\}$, let $y \in P_{\delta} x$. Then $x \in \hat{x}_{y}$ so $y \subset x_{y} \subset x$. Hence $v(y) \subset x_{y} \subset x$. Therefore, $x \in C(v)$ and $C(v) \in \triangle^{\delta} F S F_{\kappa \lambda}$.

For the proof of part two, let $C \in \triangle^{\delta} F_{\kappa \lambda}^{\xi}$, for any regular $\xi<\delta$. Without loss of generality, assume

$$
C=\triangle^{\delta}\left\{C\left(v_{y}\right): y \in P_{\delta} \lambda \text { and } v_{y}: P_{\xi} \lambda \longrightarrow P_{\kappa} \lambda\right\}
$$

Define $\bar{v}_{y}: P_{\delta} \lambda \longrightarrow P_{\kappa} \lambda$ by $\bar{v}_{y}(s)=\bigcup\left\{v_{y}(t): t \in P_{\xi} s\right\}$. Now

$$
\triangle^{\delta}\left\{C\left(\bar{v}_{y}\right): y \in P_{\delta} \lambda\right\} \in F_{\kappa \lambda}^{\delta},
$$

since $F_{\kappa \lambda}^{\delta}$ is $\delta$-normal. Given $x \in \triangle^{\delta}\left\{C\left(\bar{v}_{y}\right): y \in P_{\delta} \lambda\right\}, y \in P_{\delta} x$ and $t \in P_{\xi} x$, then for any $s \in P_{\delta} x$ such that $t \subset s$, yields $v_{y}(t) \subset \bar{v}_{y}(s) \subset x$. Hence $x \in C$. Therefore, $C \in F_{\kappa \lambda}^{\delta}$.

Finally, let $v: P_{\delta} \lambda \longrightarrow P_{\kappa} \lambda$. For $y \in P_{\delta} \lambda$, define $v_{y}: P_{\xi} \lambda \longrightarrow P_{\kappa} \lambda$ by $v_{y}(t) \in C(v)$ such that $y \subset v_{y}(t)$ for every $t \in P_{\xi} \lambda$. This gives $C\left(v_{y}\right) \in F_{\kappa \lambda}^{\xi}$ for each $y \in P_{\delta} \lambda$. Let $x \in \triangle^{\delta}\left\{C\left(v_{y}\right): y \in P_{\delta} \lambda\right\}$. If $y \in P_{\delta} x$, then $x \in C\left(v_{y}\right)$. For any $t \in P_{\xi} x$, it is the case that $y \subset v_{y}(t)$ and $v_{y}(t) \in C(v)$. This gives $v(y) \subset v_{y}(t)$. But $x \in C\left(v_{y}\right)$, and hence $v(y) \subset v_{y}(t) \subset x$. Therefore $x \in$ $C(v)$ and $C(v) \in \triangle^{\delta} F_{\kappa \lambda}^{\xi}$.

Corollary 15. $F_{\kappa \lambda}^{\delta}$ is the minimal $\delta$-normal, fine, $\kappa$-complete filter on $P_{\kappa} \lambda$. Proof. Let $F$ be any $\delta$-normal, fine, $\kappa$-complete filter on $P_{\kappa} \lambda$. Then $F S F_{\kappa \lambda} \subset$ $F$ and $\triangle^{\delta} F S F_{\kappa \lambda} \subset \triangle^{\delta} F=F$, giving $F_{\kappa \lambda}^{\delta} \subset F$. 
This section looks at the notions of stationarity and saturation related to the hierarchy of filters, $F_{\kappa \lambda}^{\delta}$ for $\delta<\bar{\delta}$ regular and presents some well-known results in this context.

Assume $\kappa$ is inaccessible for the remainder of this section. The filters, $D C F_{\kappa \lambda}^{\delta}$ for $\delta<\bar{\delta}$ and their dual ideals $\left(D C F_{\kappa \lambda}^{\delta}\right)^{*}$, are natural extensions of $C F_{\kappa \lambda}$ and the nonstationary ideal, $N S_{\kappa \lambda}$. Since $F_{\kappa \lambda}^{\delta}=D C F_{\kappa \lambda}^{\delta}$ it holds that $\left(F_{\kappa \lambda}^{\delta}\right)^{+} \subset C F_{\kappa \lambda}^{+}$.

Definition 16. Say $S \subset P_{\kappa} \lambda$ is $\delta$-stationary if $A \cap S \neq \varnothing$, for all $A$ in $F_{\kappa \lambda}^{\delta}$. That is, $S$ is $\delta$-stationary if and only if $S \in\left(F_{\kappa \lambda}^{\delta}\right)^{+}$.

The following theorem is in the spirit of [Me, Corollary 16], and characterizes $\delta$-stationary sets in terms of " $\delta$-regressive" functions.

Theorem 17. For $\delta<\bar{\delta}, S \subset P_{\kappa} \lambda$ is $\delta$-stationary if and only if for every $f: P_{\kappa} \lambda \longrightarrow P_{\delta} \lambda$ such that $f(x) \in P_{\delta} x, \forall x \in S$ (say $f$ is $\delta$-regressive over $S$ ), there exists an unbounded subset of $S$ where $f$ is constant.

Proof. One direction follows directly from the fact that $F_{\kappa \lambda}^{\delta}$ is $\delta$-normal.

For the other direction, assume that $S$ is not $\delta$-stationary. Let $v: P_{\delta} \lambda \longrightarrow$ $P_{\kappa} \lambda$ be such that $C(v) \subset P_{\kappa} \lambda-S$. Define $f: S \longrightarrow P_{\delta} \lambda$ by $f(x) \in P_{\delta} x$ such that $v(f(x)) \nsubseteq x$. But this leads to a contradiction, since $f^{-1}(\{y\}) \cap S$ cannot be unbounded for any $y \in P_{\delta} \lambda$.

Recall that an ideal $I$ is said to be $\mu$-saturated if whenever $\left\{A_{\gamma}: \gamma<\nu\right\} \subset$ $I^{+}$such that $A_{\gamma} \cap A_{\gamma^{\prime}} \in I$ for $\gamma \neq \gamma^{\prime}$, then $\nu<\mu$.

The next theorem gives a characterization of saturation for $\delta$-normal ideals on $P_{\kappa} \lambda$ in terms of their possible extensions by $\delta$-normal ideals on $P_{\kappa} \lambda$. This type of characterization was first used in [B-T-W] for normal ideals on a cardinal $\kappa$.

Given an ideal $I$ on $P_{\kappa} \lambda$, let

$$
I \mid A=\left\{B \subset P_{\kappa} \lambda: A \cap B \in I\right\} .
$$

This is the ideal generated by $I$ and $P_{\kappa} \lambda-A$. Observe that if $A \in I^{*}$, then $I \mid A=I$ and if $A \in I$ then $I \mid A=P\left(P_{\kappa} \lambda\right)$. Hence $I \mid A$ is a proper extension of $I$ when $A \in I^{+}-I^{*}$.

Theorem 18. Let $\delta<\kappa . A \delta$-normal, fine ideal I on $P_{\kappa} \lambda$ is $\left(\lambda^{<\delta}\right)^{+}$-saturated if and only if $I \mid A$ for $A \in I^{+}-I^{*}$ are the only $\delta$-normal ideals on $P_{\kappa} \lambda$ which properly extend $I$.

Proof. The proof follows the argument of [B-T-W] with straightforward modifications.

Mahlo's operation: for $A \subset \kappa$,

$$
M(A)=\left\{\alpha<\kappa: c f(\alpha)>\omega \text { and } A \cap \alpha \notin N S_{\alpha}\right\}
$$

led to the notion of an $M$-ideal, which plays a major role in gaining results on splitting stationary sets on $\kappa$ (see [B-T-W]), and on $P_{\kappa} \lambda$ (see [Jo]). In the latter case, Johnson first generalized the Mahlo operation to $P_{\kappa} \lambda$ as follows: for $A \subset P_{\kappa} \lambda$

$$
M(A)=\left\{x \in A: x \cap \kappa \text { is a weak. inacc. and } A \cap P_{x \cap \kappa} x \in N S_{x \cap \kappa, x}^{+}\right\} .
$$


An $M$-ideal is an ideal $I$, which satisfies: $M(A) \in I^{*}$ if and oniy if $A \in I^{*}$.

Theorem 18, in combination with the following statement provide the base for the results on splitting stationary subsets in the two papers mentioned in the preceding paragraph: If $I$ is an $M$-ideal then, for any stationary subset $A$, $I \neq N S \mid A$ (where NS stands for the nonstationary ideal on $\kappa$ or $P_{\kappa} \lambda$ ). In the latter case, the fact that the closed unbounded filter on $P_{\kappa} \lambda$ can be replaced by the filter generated by closure sets of functions $v: P_{3} \lambda \longrightarrow P_{\kappa} \lambda$ provides the key to the proof of this statement.

A variation of Johnson's generalization of the Mahlo operation to $P_{\kappa} \lambda$ is presented, which is directly analogous to the way Jech generalized the diamond principle to $P_{\kappa} \lambda$ in [Je2].

Definition 19. For $A \subset P_{\kappa} \lambda$ and $\delta<\bar{\delta}$,

$$
M^{\delta}(A)=\left\{x \in A:|x| \text { is weak. inacc., }|x|>\delta \text { and } A \cap P_{|x|} x \in\left(F_{|x|, x}^{\delta}\right)^{+}\right\} .
$$

And for $I$ a $\delta$-normal ideal, say $I$ is an $M^{\delta}$-ideal if $M^{\delta}(A) \in I^{*}$ whenever $A \in I^{*}$.

The usefulness of the filter generated by closure sets of functions $v: P_{\delta} \lambda \longrightarrow$ $P_{\kappa} \lambda$ will be used to extend the statement mentioned in the preceding paragraph to this context.

Theorem 20. If $I$ is an $M^{\delta}$-ideal on $P_{\kappa} \lambda$ then, for any $A \in\left(F_{\kappa \lambda}^{\delta}\right)^{+}, I \neq$ $\left(F_{\kappa \lambda}^{\delta}\right)^{*} \mid A$.

Proof. Suppose $I$ is an $M^{\delta}$-ideal and $I=\left(F_{\kappa \lambda}^{\delta}\right)^{*} \mid A$ for some $A \in\left(F_{\kappa \lambda}^{\delta}\right)^{+}$. Then there exists a $v: P_{\delta} \lambda \longrightarrow P_{\kappa} \lambda$ such that $C(v) \cap A \subseteq M^{\delta}(A)$.

Claim. There exists a $B \subset C(v)$ such that

$$
B=\left\{x \in P_{\kappa} \lambda: \forall y \in P_{\delta} x(v(y) \subset x \wedge|v(y)|<|x|)\right\}
$$

and $B \in D C F_{\kappa \lambda}^{\delta}$.

Proof of claim. The proof of this claim is a straightforward exercise and will be omitted.

Let $x \in B \cap A \subset M^{\delta}(A)$ be such that $|x|$ is minimal. Define $v_{x}: P_{\delta} x \longrightarrow$ $P_{|x|} x$ by $v_{x}(y)=v(y)$. This holds since $x \in C(v)$ and $y \in P_{\delta} x$, and hence $v_{x}(y) \subset x$. Also, $x \in B$ gives $\left|v_{x}(y)\right|<|x|$ and $v_{x}(y) \in P_{|x|} x$. But

$$
C\left(v_{x}\right) \subset C(v) \cap P_{|x|} x
$$

since first: $v_{x}$ assumes values in $P_{|x|} x$ so $C\left(v_{x}\right) \subset P_{|x|} x$; and if $y \in C\left(v_{x}\right)$ and $z \in P_{\delta} y \subset P_{\delta} x$, then $v(z)=v_{x}(z) \subset y$; hence, $y \in C(v)$. Next, let $B_{x} \subset C\left(v_{x}\right)$ such that

$$
B_{x}=\left\{y \in P_{|x|} x: \forall z \in P_{\delta} y\left(v_{x}(z) \subset y \wedge\left|v_{x}(z)\right|<|y|\right)\right\} .
$$

Now $B_{x} \in F_{|x|, x}^{\delta}$ and hence $B \cap P_{|x|} x \in F_{|x|, x}^{\delta}$. But $x \in M^{\delta}(A)$, so $A \cap P_{|x|} x \in$ $\left(F_{|x|, x}^{\delta}\right)^{+}$. But this means that there exists $y \in B \cap A \cap P_{|x|} x$, contradicting the choice of $x$.

The theorems of this section are not meant to be exhaustive, but rather they are meant to suggest that a great deal of what can be done for $C_{\kappa \lambda}$ can in turn be done for $F_{\kappa \lambda}^{\delta}$. Another example of this point comes from analyzing the work of Matsubara in [Mat]. A consequence of Matsubara's technique is that $P_{\kappa} \lambda$ splits into $\lambda^{<\kappa}$ many disjoint $\delta$-stationary subsets. 


\section{REFERENCES}

[B-T-W] J. E. Baumgartner, A. D. Taylor, and S. Wagon, On splitting stationary subsets of large cardinals, J. Symbolic Logic 42 (1977), 203-214.

[Ca] D. M. Carr, The minimal normal filter on $P_{\kappa} \lambda$, Proc. Amer. Math. Soc. 86 (1982), 316-320.

[Je1] T. J. Jech, Set theory, Academic Press, 1978.

[Je2] - Some combinatorial problems concerning uncountable cardinals, Ann. of Math. Logic 5 (1973), 165-198.

[Jo] C. A. Johnson, On saturated ideals and $P_{\kappa} \lambda$, Fund. Math. 129 (1988), 215-221.

[Mag] M. Magidor, Combinatorial characterization of supercompact cardinals, Proc. Amer. Math. Soc. 42 (1974), 279-285.

[Mat] Y. Matsubara, Splitting $P_{\kappa} \lambda$ into stationary subsets, J. Symbolic Logic 53 (1988), 385-389.

[Me] T. K. Menas, On strong compactness and supercompactness, Ann. of Math. Logic 7 (1974), 327-359.

[Mi] R. J. Mignone, A direct weakening of normality for filters (to appear).

Mathematical Sciences Research Institute, Berkeley, California 94720

Current address: Department of Mathematics, The College of Charleston, Charleston, South Carolina 29424 